Discrete Comput Geom 36:273-281 (2006)

DOI: $10.1007 / \mathrm{s} 00454-006-1235-1$

\title{
How Neighborly Can a Centrally Symmetric Polytope Be?
}

\author{
Nathan Linial ${ }^{1}$ and Isabella Novik ${ }^{2}$ \\ ${ }^{1}$ Institute of Computer Science, Hebrew University, \\ Jerusalem 91904, Israel \\ nati@cs.huji.ac.il \\ ${ }^{2}$ Department of Mathematics, University of Washington, \\ Box 354350, Seattle, WA 98195-4350, USA \\ novik@math.washington.edu \\ Communicated by Günter Ziegler
}

\begin{abstract}
We show that there exist $k$-neighborly centrally symmetric $d$-dimensional polytopes with $2(n+d)$ vertices, where

$$
k(d, n)=\Theta\left(\frac{d}{1+\log ((d+n) / d)}\right) .
$$
\end{abstract}

We also show that this bound is tight.

\section{Introduction}

In this paper we study the following question: How neighborly can a centrally symmetric polytope be as a function of its dimension and the number of vertices? Let us recall the basic definitions: A polytope $P \subset \mathbb{R}^{d}$ is centrally symmetric (cs, for short) if for every $x \in P,-x$ belongs to $P$ as well. A cs polytope $P$ is called $k$-neighborly if every set of $k$ of its vertices, no two of which are antipodes, is the vertex set of a face of $P$.

It is well-know that a general (non-cs) $d$-dimensional polytope with at least $d+$ 2 vertices can be at most $\lfloor d / 2\rfloor$-neighborly, and this bound is attained for instance by $d$-dimensional cyclic polytopes [18, Example 0.6]. In contrast to the general case, the neighborliness of cs polytopes appears to be quite restricted and not sufficiently understood. A cs $d$-polytope with at least $2(d+2)$ vertices cannot be more than $\mathrm{L}(d+$ $1) / 3\rfloor$-neighborly. The case $d=4$ of this statement was observed by Grünbaum [8, p. 116] in 1967, whereas the general case is due to McMullen and Shephard [12]. These 
authors have also conjectured that a cs $d$-polytope with $2(d+n)$ vertices cannot be more than $\lfloor(d+n-1) /(n+1)\rfloor$-neighborly for all $n \geq 3$. Their conjecture was refuted by Halsey [9] and then by Schneider [15], but only for $d \gg n$. Namely, Schneider's theorem asserts that

$$
\liminf _{d \rightarrow \infty} \frac{k(d, n)}{d+n} \geq 0.2390,
$$

where $k(d, n)$ denotes the largest integer $k$ such that there exists a $k$-neighborly cs $d$ polytope with $2(n+d)$ vertices.

A particularly interesting case is when $k(d, n)=1$. Namely, given $d$, how large must $n$ be so that a cs $d$-polytope with $\geq 2(n+d)$ vertices cannot be even 2-neighborly? That a cs $d$-polytope with a sufficiently large number of vertices $\left(\approx(d / 2)^{d / 2}\right)$ indeed cannot be 2-neighborly was verified by Burton [3]. The McMullen-Shephard conjecture posits that this already holds for $n=d-2$, but this turns out to be incorrect, since we show that this critical $n$ is exponential in $d$. No other facts on $k(d, n)$ appear to exist in the literature.

Here we compute the correct asymptotics of $k(d, n)$, thus establishing an extension of Schneider's and Burton's results. Throughout the paper we set $m:=n+d$.

\section{Theorem 1.1.}

$$
\frac{C_{1} d}{1+\log (m / d)} \leq k(d, n) \leq 1+\frac{C_{2} d}{1+\log (m / d)},
$$

where $C_{1}, C_{2}>0$ are absolute constants independent of $d$ and $n$. In particular, there exists a cs d-polytope with $4 d$ vertices that is at least $d / 400$-neighborly.

Theorem 1.1 provides the correct asymptotic answer for the above problem and shows that the largest number of vertices in a 2-neighborly cs $d$-polytope is $e^{\Theta(d)}$. In fact we can say a bit more here:

Theorem 1.2. $k\left(d, 2^{d-1}+1-d\right)=1$. In other words, a 2-neighborly cs $d$-polytope has at most $2^{d}$ vertices.

We prove Theorem 1.2 as a warm-up for the proof of the upper bound in Theorem 1.1. These proofs appear in Section 2. The former result is a consequence of the DanzerGrünbaum theorem on the number of vertices of antipodal polytopes [5] (a more readily accessible source for a proof of this theorem is Aigner and Ziegler's beautiful book [1, Chapter 14]) and the observation that every 2-neighborly cs polytope is antipodal. For the latter result we establish a certain modification of the Danzer-Grünbaum argument.

The proof of the lower bound in Theorem 1.1 is based on studying the cs transforms of cs polytopes introduced in [12] and on a theorem due to Garnaev and Gluskin [7]. This theorem concerns the intersection of the $m$-dimensional octahedron $B_{1}^{m}$ with any $n$-dimensional subspace of $\mathbb{R}^{m}$. The question is how close to an $n$-dimensional Euclidean ball such an intersection can be. We outline the necessary background on cs transforms in Section 3. The verification of the lower bound in Theorem 1.1 and the statement of the Garnaev-Gluskin theorem are provided in Section 4. The proof of the Garnaev-Gluskin 
result and hence also of Theorem 1.1 is probabilistic in nature: it does not give an explicit construction of neighborly cs polytopes, but rather shows that they form a set of positive probability in a certain probability space. Indeed, it is an interesting open question to find explicit constructions of highly neighborly cs polytopes that meet the lower bound we prove.

Remark 1.3. After this paper was submitted, it was brought to our attention that Rudelson and Vershynin [14] have independently established, using basically the same technique, a dual version of the lower bound part of Theorem 1.1. Namely, they showed that there is a constant $C$ such that for all $f, r$, and $d \geq C r \log (f / r)$, there exists a cs $d$-polytope with $f$ facets and the maximal number $2^{r}\left(\begin{array}{l}f \\ r\end{array}\right)$ of $(d-r)$-faces. Their work was done in the context of coding theory and extended a result by Donoho [6] asserting that for large $d$, the orthogonal projection of the $2 d$-dimensional octahedron $B_{1}^{2 d}$ on a uniform random $d$-dimensional subspace is with high probability at least $0.089 d$ neighborly (compare with the "in particular" part of Theorem 1.1). These emerging strong connections between two seemingly unrelated areas-error-correcting codes and neighborliness of cs polytopes-are fascinating. This is a very active area of current research, see, e.g., [17].

\section{The Upper Bound on $k(d, n)$}

The goal of this section is to verify Theorem 1.2 and the upper bound in Theorem 1.1. To this end, recall from [8, p. 420] that two vertices $v$ and $w$ of a $d$-polytope $Q \subset \mathbb{R}^{d}$ are an antipodal pair if there exist two parallel (distinct) supporting hyperplanes of $Q$, one of which contains $v$ and the other one contains $w$. A $d$-polytope $Q$ is called an antipodal polytope if every two of its vertices form an antipodal pair. The theorem due to Danzer and Grünbaum [5] (see also Chapter 14 of [1]) asserts that an antipodal $d$-polytope cannot have more than $2^{d}$ vertices. Theorem 1.2 is then an immediate consequence of their result and the following observation.

Lemma 2.1. Every 2-neighborly cs d-polytope is an antipodal polytope.

Proof. Consider two vertices $v$ and $w$ of a 2-neighborly cs $d$-polytope $P$. We show that they form an antipodal pair. There are two possible cases: either $v=-w$ or $v \neq-w$. In the first case, let $H$ be any hyperplane satisfying $H \cap P=\{v\}$. (Such an $H$ exists since $v$ is a vertex of $P$.) Then $H$ is a supporting hyperplane of $P$ that contains $v$, while $-H:=\left\{x \in \mathbb{R}^{d}:-x \in H\right\}$ is a supporting hyperplane of $P$ that contains $w=-v$. In the second case, consider the set $E=\operatorname{conv}\{v,-w\}$. Since $P$ is 2-neighborly, $E$ is an edge of $P$, and so there exists a hyperplane $H$ such that $H \cap P=E$. Then $H$ is a supporting hyperplane of $P$ that contains $v$, while $-H$ is a supporting hyperplane of $P$ that contains the edge $E^{\prime}=-E=\operatorname{conv}\{-v, w\}$, and hence also the vertex $w$.

The proof of the upper bound in Theorem 1.1 is obtained by a certain modification of the Danzer-Grünbaum argument and is based on the following simple combinatorial result. (We denote by $\left(\begin{array}{c}{[m]} \\ s\end{array}\right)$ the family of all $s$-subsets of $[m]:=\{1, \ldots, m\}$.) 
Lemma 2.2. For every two integers $s$ and $m$ with $s \leq m / 2$, there exists a family $\mathcal{F} \subset\left(\begin{array}{c}{[m]} \\ s\end{array}\right)$ of size $\geq(C m / s)^{s / 2}$ such that $|A \cap B| \leq s / 2$ for every two distinct $A, B \in \mathcal{F}$. Here $C>0$ is an absolute constant.

Proof. We construct $\mathcal{F}$ by a simple greedy argument. Let $\mathcal{F} \subset\left(\begin{array}{c}{[m]} \\ s\end{array}\right)$ be an inclusionmaximal family satisfying the condition $|A \cap B| \leq s / 2$ for all $A, B \in \mathcal{F}$. We claim that $|\mathcal{F}| \geq(C m / s)^{s / 2}$. Indeed, for a set $A \in \mathcal{F}$ define the "forbidden collection" $A^{f}:=$ $\left\{B \in\left(\begin{array}{c}{[m]} \\ s\end{array}\right):|A \cap B|>s / 2\right\}$. Then

$$
\left|A^{f}\right|=\sum_{k=1}^{\lceil s / 2\rceil}\left(\begin{array}{c}
s \\
\lfloor s / 2\rfloor+k
\end{array}\right)\left(\begin{array}{c}
m-s \\
\lceil s / 2\rceil-k
\end{array}\right)<\left(\begin{array}{c}
m \\
\lfloor s / 2\rfloor
\end{array}\right) \sum_{k=1}^{\lceil s / 2\rceil}\left(\begin{array}{c}
s \\
\lfloor s / 2\rfloor+k
\end{array}\right)<\left(\begin{array}{c}
m \\
\lfloor s / 2\rfloor
\end{array}\right) 2^{s},
$$

and so

$$
\left|\bigcup_{A \in \mathcal{F}} A^{f}\right| \leq|\mathcal{F}| \cdot\left(\begin{array}{c}
m \\
\lfloor s / 2\rfloor
\end{array}\right) 2^{s} \leq|\mathcal{F}| \cdot 2^{s}\left(\frac{e m}{s / 3}\right)^{s / 2}=|\mathcal{F}| \cdot\left(\frac{12 e m}{s}\right)^{s / 2}
$$

(where the penultimate step follows from the inequality $\left.\left(\begin{array}{c}m \\ t\end{array}\right) \leq(\mathrm{em} / t)^{t}\right)$. On the other hand, the maximality of $\mathcal{F}$ implies that $\mathcal{F} \cup\left(\bigcup_{A \in \mathcal{F}} A^{f}\right)=\left(\begin{array}{c}{[m]} \\ s\end{array}\right)$. Hence $|\mathcal{F}| \cdot(1+$ $\left.(12 e m / s)^{s / 2}\right) \geq\left(\begin{array}{l}m \\ s\end{array}\right)$ which together with the inequality $\left(\begin{array}{c}m \\ s\end{array}\right) \geq(\mathrm{m} / s)^{s}$ yields the result.

We are now in a position to prove the upper bound in Theorem 1.1 asserting that $k(d, n) \leq 1+C_{2} d /(1+\log (m / d))$ for some absolute constant $C_{2}>0$. To do so consider a cs $d$-polytope $P$ on the vertex set $V=\left\{v_{1},-v_{1}, \ldots, v_{m},-v_{m}\right\}$ that is $2 s$ neighborly. Thus $2 s \leq d \leq m$. Let $\mathcal{F} \subset\left(\begin{array}{c}{[m]} \\ s\end{array}\right)$ be a family from Lemma 2.2. For each set $A \in \mathcal{F}$, define

$$
P_{A}:=P+2 / s \sum_{i \in A} v_{i} \subseteq P+2 P=3 P
$$

to be a translate of $P$, where "+" denotes Minkowski addition.

We claim that the polytopes $P_{A}, A \in \mathcal{F}$, have pairwise disjoint interiors, whence

$$
|\mathcal{F}| \cdot \operatorname{vol}(P)=\sum_{A \in \mathcal{F}} \operatorname{vol}\left(P_{A}\right) \leq \operatorname{vol}(3 P)=3^{d} \cdot \operatorname{vol}(P) .
$$

It follows that

$$
3^{d} \geq|\mathcal{F}| \geq(\mathrm{Cm} / \mathrm{s})^{s / 2} .
$$

Thus $d \cdot \log 3 \geq s / 2 \cdot \log (C m / s) \geq \Omega(s \cdot \log (m / d))$, as claimed.

We turn to show that for any two distinct $A, B \in \mathcal{F}$ the sets $P_{A}$ and $P_{B}$ have disjoint interiors. The symmetric difference $R:=(A-B) \cup(B-A) \subseteq[m]$ has cardinality $s \leq|R| \leq 2 s$ since $|A|=|B|=s$ and $|A \cap B| \leq s / 2$. Therefore, the fact that $P$ is $2 s$-neighborly implies that $\left\{-v_{i}: i \in A-B\right\} \cup\left\{v_{j}: j \in B-A\right\} \subset V$ is the vertex set, $\operatorname{vert}(F)$, of some proper face $F$ of $P$. We want to rule out the possibility that 
$x+(2 / s) \sum_{i \in A} v_{i}=y+(2 / s) \sum_{i \in B} v_{i}$ for some $x, y \in \operatorname{int}(P)$. Indeed,

$$
\begin{aligned}
\frac{1}{2}\left[x+\frac{2}{s} \sum_{i \in A} v_{i}\right]-\frac{1}{2}\left[y+\frac{2}{s} \sum_{j \in B} v_{j}\right] & =\frac{x-y}{2}-\frac{1}{s}\left[\sum_{i \in A-B}\left(-v_{i}\right)+\sum_{j \in B-A} v_{j}\right] \\
& =\frac{x-y}{2}-\frac{|R|}{s} \sum_{v \in \operatorname{vert}(F)} \frac{1}{|R|} v \neq 0,
\end{aligned}
$$

since $(x-y) / 2$ is an interior point of $P$, while $(|R| / s) \sum_{v}(1 /|R|) v$ is the $|R| / s \geq 1$ multiple of a boundary point of $P$, namely of the barycenter of $F$. The conclusion follows.

\section{Centrally Symmetric Transforms}

Throughout the rest of the paper we denote the standard scalar product on $\mathbb{R}^{n}$ by $\langle-,-\rangle$. The abbreviations int, rel int, and conv stand for the interior, relative interior, and convex hull, respectively.

Following [12], we define a centrally symmetric set (cs set, for short) as a finite spanning subset of $\mathbb{R}^{d}$ of the form $V=\left\{v_{1},-v_{1}, \ldots, v_{m},-v_{m}\right\}$. The construction described in [12] associates with a cs set $V=\left\{ \pm v_{1}, \ldots, \pm v_{m}\right\} \subset \mathbb{R}^{d}$ another cs set $\bar{V}=\left\{ \pm \bar{v}_{1}, \ldots, \pm \bar{v}_{m}\right\} \subset \mathbb{R}^{m-d}=\mathbb{R}^{n}$ called the cs transform of $V$. This operation possesses the following properties:

(1) Let $\bar{V}=\left\{ \pm \bar{v}_{1}, \ldots, \pm \bar{v}_{m}\right\} \subset \mathbb{R}^{n}$ be a cs set. Then $\bar{V}$ is a cs transform of the vertex set $V$ of a cs $d$-polytope $P$ with $2 m$ vertices if and only if

$$
\bar{v}_{i} \in \operatorname{int} \text { conv }\left\{\sum_{l \in[m]-\{i\}} \varepsilon_{l} \bar{v}_{l}: \varepsilon_{l} \in\{1,-1\}\right\} \quad \text { for all } i=1, \ldots, m .
$$

(2) More generally, if $V=\left\{ \pm v_{1}, \ldots, \pm v_{m}\right\} \subset \mathbb{R}^{d}$ is the vertex set of a cs $d$-polytope $P$ and $\bar{V}$ is a cs transform of $V$, then the set $\left\{\delta_{i} v_{i}: i \in I\right\} \subset V$ (where $\delta_{i} \in\{1,-1\}$, $i \in I$, are fixed signs and $\left.I=\left\{i_{1}<\cdots<i_{k}\right\} \subseteq[m]\right)$ is the vertex set of a face of $P$ if and only if

$$
\sum_{i \in I} \delta_{i} \bar{v}_{i} \in \operatorname{relint} \operatorname{conv}\left\{\sum_{l \in[m]-I} \varepsilon_{l} \bar{v}_{l}: \varepsilon_{l} \in\{1,-1\}\right\} .
$$

Thus if $V=\left\{ \pm v_{1}, \ldots, \pm v_{m}\right\} \subset \mathbb{R}^{d}$ is a cs set, then its subset $\left\{\delta_{i} v_{i}: i \in I\right\}$ (for some $\delta_{i} \in\{1,-1\}$ and $I=\left\{i_{1}<\cdots<i_{k}\right\} \subseteq m$ ) fails to be the vertex set of a face of the cs polytope $P:=\operatorname{conv} V$ if and only if there exists $u \in \mathbb{R}^{n}$ such that

$$
0 \neq\left\langle\sum_{i \in I} \delta_{i} \bar{v}_{i}, u\right\rangle \geq\left\langle\sum_{l \in[m]-I} \pm \bar{v}_{l}, u\right\rangle \quad \text { for all choices of signs, }
$$

that is, if and only if

$$
0 \neq \sum_{i \in I}\left\langle\delta_{i} \bar{v}_{i}, u\right\rangle \geq \sum_{l \in[m]-I}\left|\left\langle\bar{v}_{l}, u\right\rangle\right|
$$


We call a subset $\left\{\bar{v}_{i}: i \in I\right\}$ of $\bar{V}_{+}:=\left\{\bar{v}_{1}, \ldots, \bar{v}_{m}\right\} \in \mathbb{R}^{n}$ dominant if there exists $0 \neq u \in \mathbb{R}^{n}$ such that

$$
\sum_{i \in I}\left|\left\langle\bar{v}_{i}, u\right\rangle\right| \geq \sum_{l \in[m]-I}\left|\left\langle\bar{v}_{l}, u\right\rangle\right|, \quad \text { or, equivalently, } \quad \sum_{i \in I}\left|\left\langle\bar{v}_{i}, u\right\rangle\right| \geq \frac{1}{2} \sum_{l=1}^{m}\left|\left\langle\bar{v}_{l}, u\right\rangle\right| .
$$

Since $\sum_{i \in I}\left\langle\delta_{i} \bar{v}_{i}, u\right\rangle \leq \sum_{i \in I}\left|\left\langle\bar{v}_{i}, u\right\rangle\right|$ for $\delta_{i}= \pm 1$, and since equality is attained for a certain choice of signs, we obtain the following criterion.

Lemma 3.1. A cs set $\bar{V}=\left\{ \pm \bar{v}_{1}, \ldots, \pm \bar{v}_{m}\right\} \subset \mathbb{R}^{n}$ is a cs transform of the vertex set of a k-neighborly cs d-polytope with $2 m=2(n+d)$ vertices if and only if the set $\bar{V}_{+}:=\left\{\bar{v}_{1}, \ldots, \bar{v}_{m}\right\}$ does not contain dominant subsets of size $k$.

Thus to prove lower bounds on $k(d, n)$ it suffices to construct vector configurations spanning $\mathbb{R}^{n}$ that do not contain small dominant subsets. This is done in the following section.

\section{Vector Configurations without Small Dominant Subsets}

For a vector $x=\left(x_{1}, \ldots, x_{m}\right) \in \mathbb{R}^{m}$ its $l_{1}^{m}$ and $l_{2}^{m}$ norms are defined as $\|x\|_{1}:=\sum_{i=1}^{m}\left|x_{i}\right|$ and $\|x\|_{2}:=\sqrt{\sum_{i=1}^{m}\left|x_{i}\right|^{2}}$, respectively. Thus the unit ball of $l_{1}^{m}$ is the $m$-dimensional octahedron $B_{1}^{m}:=\left\{x \in \mathbb{R}^{m}: \sum_{i=1}^{m}\left|x_{i}\right| \leq 1\right\}$, while the unit ball of $l_{2}^{m}$ is the $m$ dimensional Euclidean ball $B_{2}^{m}=\left\{x \in \mathbb{R}^{m}: \sum_{i=1}^{m} x_{i}^{2} \leq 1\right\}$. The theorem due to Garnaev and Gluskin [7] (see [11] for a simplified proof) quantifies the extent to which the intersection of $B_{1}^{m}$ with an $n$-dimensional subspace of $\mathbb{R}^{m}$ can be close to $B_{2}^{n}$. It asserts that for any natural numbers $d$ and $n$ there exists a subspace $L^{d}$ of $\mathbb{R}^{m}$ of codimension $d$ (equivalently, of dimension $m-d=n$ ), such that

$$
\|x\|_{2} \leq \widetilde{C} \sqrt{\frac{1+\log (m / d)}{d}} \cdot\|x\|_{1} \quad \text { for all } \quad x \in L^{d}
$$

where $\widetilde{C}$ is an absolute constant independent of $d$ and $m$. (In fact, the set of such subspaces has a positive measure in the Grassmannian manifold $G_{m-d, m}$ of all codimension $d$ subspaces of $\mathbb{R}^{m}$ endowed with the normed unitary invariant measure.) In the following we refer to such subspace $L^{d}$ as a Garnaev-Gluskin subspace. A weaker version of this theorem, with $(1+\log (m / d))^{3 / 2}$ instead of $(1+\log (m / d))^{1 / 2}$, had been shown earlier by Kašin [10].

Since $L^{d} \subset \mathbb{R}^{m}$ is an $n$-dimensional space, there is a linear injective map $T: \mathbb{R}^{n} \rightarrow$ $\mathbb{R}^{m}$ whose image is $L^{d}$. Let $A$ be the $m \times n$ matrix representing this map, and let $\bar{v}_{1}, \ldots, \bar{v}_{m} \in \mathbb{R}^{n}$ be the rows of this matrix. Then for every $0 \neq u \in \mathbb{R}^{n}, T(u)$ is a non-zero element of $L^{d}$ whose $i$ th coordinate is given by $\left\langle\bar{v}_{i}, u\right\rangle$ (for $i=1, \ldots, m$ ). 
Hence for every $k$-element subset $\left\{\bar{v}_{i}: i \in I\right\}$ of $\left\{\bar{v}_{1}, \cdots, \bar{v}_{m}\right\}$ and for every $0 \neq u \in \mathbb{R}^{n}$, we have

$$
\begin{aligned}
\sum_{i \in I}\left|\left\langle\bar{v}_{i}, u\right\rangle\right| & \leq \sqrt{k} \cdot \sqrt{\sum_{i \in I}\left\langle\bar{v}_{i}, u\right\rangle^{2}} \quad \text { (by the Cauchy-Schwarz inequality) } \\
& \leq \sqrt{k} \cdot\|T(u)\|_{2} \leq \sqrt{k} \cdot \widetilde{C} \cdot \sqrt{\frac{1+\log (m / d)}{d}} \cdot\|T(u)\|_{1} \quad \text { (by }(1) \\
& =\sqrt{k} \cdot \widetilde{C} \cdot \sqrt{\frac{1+\log (m / d)}{d}} \cdot \sum_{l=1}^{m}\left|\left\langle\bar{v}_{l}, u\right\rangle\right|<\frac{1}{2} \sum_{l=1}^{m}\left|\left\langle\bar{v}_{l}, u\right\rangle\right|
\end{aligned}
$$

as long as $k<\left(4 \widetilde{C}^{2}\right)^{-1} \cdot d /(1+\log (m / d))$. Therefore we infer the following result.

Lemma 4.1. Let $L^{d}$ be a Garnaev-Gluskin subspace of $\mathbb{R}^{m}$. Denote by $\bar{V}_{+}=\left\{\bar{v}_{1}, \cdots, \bar{v}_{m}\right\} \subset \mathbb{R}^{n}$ the set of rows of the matrix representing a map $T: \mathbb{R}^{n} \rightarrow \mathbb{R}^{m}$ whose image is $L^{d}$. Then $\bar{V}_{+}$does not contain dominant subsets of size smaller than $\left\lceil\left(4 \widetilde{C}^{2}\right)^{-1} \cdot d /(1+\log (m / d))\right\rceil$.

The lower bound in Theorem 1.1 asserting that for every $n$ and $d$ there exists a cs $d$-polytope with $2 m=2(n+d)$ vertices that is $\Omega(d /(1+\log (m / d)))$-neighborly is then an immediate corollary of Lemmas 3.1 and 4.1.

To obtain an estimate on the constant for the $n=d$ case (the "in particular" part of Theorem 1.1), we use the following result essentially due to Kašin (see p. 21 of [2]) asserting that there exists an orthogonal transformation $U$ of $\mathbb{R}^{d}$ such that

$$
\|x\|_{2} \leq \frac{4 R^{2}}{\sqrt{d}}\left(\left\|U^{-1} x\right\|_{1}+\|x\|_{1}\right) \quad \text { for all } \quad x \in \mathbb{R}^{d} .
$$

Here $R$ is the volume ratio of the octahedron $B_{1}^{d}$ (the notion introduced by Szarek [16]), that is,

$$
R:=\left(\frac{\operatorname{vol}\left(\sqrt{d} B_{1}^{d}\right)}{\operatorname{vol}\left(B_{2}^{d}\right)}\right)^{1 / d}=\left(\frac{2^{d} d^{d / 2}}{d !} \frac{\Gamma(d / 2+1)}{\pi^{d / 2}}\right)^{1 / d} \leq\left(\frac{2 e}{\pi}\right)^{1 / 2},
$$

where $\Gamma(\cdot)$ denotes the Gamma function.

Consider the $2 d$-element set $\bar{V}_{+}:=\left\{e_{1}, \ldots, e_{d}, U e_{1}, \ldots, U e_{d}\right\} \subset \mathbb{R}^{d}$. The calculations, completely analogous to those in the proof of Lemma 4.1, but using (2) instead of (1), imply that all dominant subsets of the set $\bar{V}_{+}$have size at least

$$
\frac{d}{2^{7} R^{4}} \stackrel{\text { by (3) }}{\geq} \frac{\pi^{2}}{2^{9} e^{2}} d \geq \frac{d}{400} .
$$

This fact together with Lemma 3.1 yields the second part of Theorem 1.1, that is, the existence of a $d / 400$-neighborly cs $d$-polytope with $4 d$ vertices. 


\section{Concluding Remarks}

We close the paper with the following remark concerning the exact value of $k(n, d)$. Let $\bar{V}_{+}=\left\{\bar{v}_{1}, \ldots, \bar{v}_{m}\right\} \subset \mathbb{R}^{n}$ be an $m$-element set that spans $\mathbb{R}^{n}$. Denote by $A=A\left(\bar{V}_{+}\right)$ the $m \times n$ matrix whose rows are the elements of $\bar{V}_{+}$. Let $T: \mathbb{R}^{n} \rightarrow \mathbb{R}^{m}$ be the map represented by $A$, and let $L^{d}=L^{d}\left(\bar{V}_{+}\right)$be the image of $T$. Clearly, $L^{d}$ has codimension $d$ in $\mathbb{R}^{m}$, and every codimension $d$ subspace of $\mathbb{R}^{m}$ arises this way.

Fix an integer $s \in[m]$ and consider the norm $\|\mid-\| \|_{s}$ on $\mathbb{R}^{m}$ defined by

$$
\left\|\left(x_{1}, \ldots, x_{m}\right) \mid\right\|_{s}:=\max \left\{\sum_{i \in \sigma}\left|x_{i}\right|: \sigma \subset[m],|\sigma|=s\right\} .
$$

(For example, $\|\mid-\| \|_{1}$ coincides with the $l_{\infty}^{m}$ norm.) Since the $i$ th coordinate of $x=$ $T(u) \in L_{d}$ equals $\left\langle\bar{v}_{i}, u\right\rangle$, it follows that $\bar{V}_{+}$does not have a dominant subset of size $s$ if and only if $\|x\|\left\|_{s}<\frac{1}{2}\right\| x \|_{1}$ for every $0 \neq x \in L^{d}$. Thus Lemma 3.1 implies that

$$
k(d, n)=\max \left\{s: c_{d}\left(l_{1},|||-|||_{s}\right)<\frac{1}{2}\right\}=\min \left\{s: c_{d}\left(l_{1},\left.\|||-\|\right|_{s}\right) \geq \frac{1}{2}\right\}-1,
$$

where $c_{d}=c_{d}\left(l_{1},\left\||-\||_{s}\right)\right.$ is the $d$ th Gelfand number,

$$
c_{d}:=\inf _{L^{d} \subset \mathbb{R}^{m}} \sup _{x \in L_{d}-\{0\}} \frac{\|x\| \|_{s}}{\|x\|_{1}} .
$$

(Here the infimum is taken over all codimension $d$ subspaces of $\mathbb{R}^{m}$.) Gelfand numbers have received a good deal of attention in Banach Space Theory [4], [13].

It is a notoriously difficult question to find explicit constructions for spaces that satisfy the conditions as in the work of Kašin, Garnaev, and Gluskin. Perhaps it is less difficult, though, to construct highly neighborly cs polytopes. Any progress on this problem would be of interest.

\section{References}

1. M. Aigner and G. M. Ziegler, Proofs from THE BOOK, third edition, Springer-Verlag, Berlin, 2004.

2. K. Ball, An elementary introduction to modern convex geometry, in Flavors of Geometry, pp. 1-58, Mathematical Sciences Research Institute Publication, 31, Cambridge University Press, Cambridge, 1997.

3. G. R. Burton, The nonneighbourliness of centrally symmetric convex polytopes having many vertices, J. Combin. Theory Ser. A 58 (1991), 321-322.

4. B. Carl and I. Stephani, Entropy, Compactness and the Approximation of Operators, Cambridge Tracts in Mathematics, 98, Cambridge University Press, Cambridge, 1990.

5. L. Danzer and B. Grünbaum, Über zwei Probleme bezüglich konvexer Körper von P. Erdös und von V. L. Klee, Math. Z. 79 (1962), 95-99.

6. D. L. Donoho, High-dimensional centrosymmetric polytopes with neighborliness proportional to dimension, available from http://www-stat.stanford.edu/ donoho/reports.html.

7. A. Yu. Garnaev and E. D. Gluskin, The widths of a Euclidean ball (in Russian), Dokl. Akad. Nauk SSSR 277(5) (1984), 1048-1052. (English translation: Soviet Math. Dokl. 30(1) (1984), 200-204.)

8. B. Grünbaum, Convex Polytopes, second edition (prepared and with a preface by V. Kaibel, V. Klee, and G. M. Ziegler), Graduate Texts in Mathematics, 221, Springer-Verlag, New York, 2003.

9. E. R. Halsey, Zonotopal complexes on the $d$-cube, Doctoral dissertation, University of Washington (1972). 
10. B. S. Kašin, The widths of certain finite-dimensional sets and classes of smooth functions (in Russian), Izv. Akad. Nauk SSSR Ser. Mat. 41(2) (1977), 334-351, 478. (English translation: Math. USSR-Izv. 11(2) (1977), 317-333.)

11. Y. Makovoz, A simple proof of an inequality in the theory of $n$-widths, in Constructive Theory of Functions (Varna, 1987), pp. 305-308, Publ. House Bulgar. Acad. Sci., Sofia, 1988.

12. P. McMullen and G. C. Shephard, Diagrams for centrally symmetric polytopes, Mathematika 15 (1968), 123-138.

13. A. Pinkus, $O n L^{1}$-Approximation, Cambridge Tracts in Mathematics, 93, Cambridge University Press, Cambridge, 1989.

14. M. Rudelson and R. Vershynin, Geometric approach to error correcting codes and reconstruction of signals, Int. Math. Res. Not. 64 (2005), 4019-4041.

15. R. Schneider, Neighbourliness of centrally symmetric polytopes in high dimensions, Mathematika 22(2) (1975), 176-181.

16. S. J. Szarek, On Kashin's almost Euclidean orthogonal decomposition of $l_{n}^{1}$, Bull. Acad. Polon. Sci. Sér. Sci. Math. Astronom. Phys. 26 (1978), 691-694.

17. Y. Zhang, A Simple Proof for Recoverability of $l_{1}$-Minimization: Go Over or Under?, Tech. Rep. TR05-09, Rice University, 2005.

18. G. M. Ziegler, Lectures on Polytopes, Graduate Texts in Mathematics, 152, Springer-Verlag, New York, 1995.

Received July 13, 2005, and in revised form November 22, 2005. Online publication April 18, 2006. 